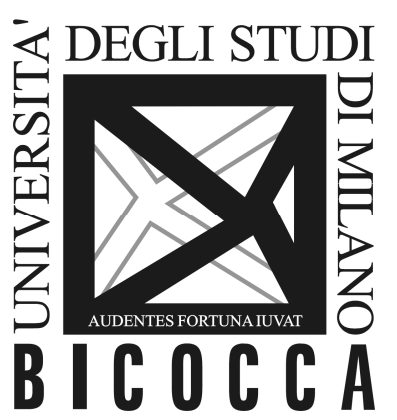

\author{
DEPARTMENT OF ECONOMICS, \\ MANAGEMENT AND STATISTICS \\ UNIVERSITY OF MILAN - BICOCCA
}

DEMS WORKING PAPER SERIES

\title{
Set them (almost) free. Discretion in electoral campaigns under asymmetric information
}

Vardan Baghdasaryan, Elena Manzoni

No. 354 - December 2016

Dipartimento di Economia, Metodi Quantitativi e Strategie di Impresa Università degli Studi di Milano - Bicocca 


\title{
Set them (almost) free. Discretion in electoral campaigns under asymmetric information*
}

\author{
Vardan Baghdasaryan ${ }^{\dagger}$ \\ Elena Manzoni ${ }^{\ddagger}$
}

December 6, 2016

\begin{abstract}
The paper analyses a model of electoral campaigning as a problem of competitive delegation. We model a situation in which there is uncertainty about what the optimal policy should be and about the extent of candidates' bias. While voters know whether the candidate is left or right wing, the bias measures the extremity of the candidate. In this environment discretion may benefit voters as it allows the elected politician to adjust his policies to the state of the world. The paper shows that the optimal set of promises must be a closed interval, whose size is decreasing in the expected bias of the candidate. An example where the set of types is finite shows that an increase in the variability of candidates' types may either increase or decrease the level of discretion granted to politicians.
\end{abstract}

Keywords: Electoral campaigns, Ideological bias, Uncertainty

JEL Classification: D72, D82

${ }^{*}$ The authors thanks Michela Cella, Giovanna Iannantuoni, Leonardo Felli, Gilat Levy, Andrea Mattozzi, Antonio Nicolò, Michele Piccione and Ronny Razin for useful comments.

${ }^{\dagger}$ American University of Armenia and Affiliate Fellow at CERGE-EI, Prague. E-mail: vbaghdasaryan@aua.am

${ }^{\ddagger}$ Corresponding author. Department of Economics, Management and Statistics, University of MilanBicocca. E-mail: elena.manzoni@unimib.it 


\section{Introduction}

"First: the reduction of the fiscal burden with total exemption for incomes up to 22 million of Italian liras per year, the reduction of the tax rate for incomes up to 200 million to $23 \%$; the reduction of the tax rate for incomes above 200 million to $33 \% .{ }^{1}$ (S. Berlusconi, 2001)

"[...] our first promise is that we will not steal money from the Italians and that we will reduce the fiscal burden below $40 \%$ of the GDP." ${ }^{2}$ (S. Berlusconi, 2008)

"What we need is a decisive government that says we are going to have a budget that will help small businesses like the ones here with a cut in Corporation Tax, saying that any new business doesn't have to pay National Insurance on the first ten new jobs, busting open state procurement so you can all bid for the state contracts, and make sure that you can make money for your businesses out of what the government does." 3 (D. Cameron, 2010)

"I want to make it absolutely crystal clear that our objective when the fiscal circumstances are right, is to lower all taxes. We want to lower all taxes. We really are the party of lower, simpler, fairer taxes look at our record in government." ${ }^{4}$ (T. Abbot, 2013)

Vagueness of electoral campaign statements is a common element of the political competition and a widely studied phenomenon in political science and economics. As the above examples show, political announcements during the electoral campaign may have different degrees of vagueness, therefore leaving different degrees of post-electoral freedom to elected politicians. This paper provides a model in which a comparative analysis of the discretion levels of political announcements can be made explicit, and the description of some of the political variables which may affect the precision of the electoral statements can be identified.

We study a model in which ambiguity of the electoral promises results in an elementary fashion from a "delegation objective": depending on how changing the environment is, the tension between rigidity and discretion that the electorate experiences may be resolved with voters having a preference for some degree of discretion. In this way they leave the politicians free to operate in a particular policy region once they are elected, so as to target their policy better to the particular state of the world. In particular, the

\footnotetext{
${ }^{1}$ In the original language: "Punto primo: Abbattimento della pressione fiscale con l'esenzione totale dei redditi fino a 22 milioni di lire annui; riduzione al 23 per cento dell'aliquota per i redditi fino a 200 milioni; riduzione al 33 per cento dell'aliquota per i redditi sopra i 200 milioni." From "Corriere della Sera", online version, $3^{\text {rd }}$ March 2008.

${ }^{2}$ In the original language: "[...] la nostra prima promessa [...] è che non metteremo mai le mani nelle tasche degli italiani e che abbasseremo la pressione fiscale sotto il 40\% del Pil."From "Corriere della Sera", online version, $29^{\text {th }}$ February 2008.

${ }^{3} \mathrm{http}: / /$ conservative-speeches.sayit.mysociety.org/speech/601487

${ }^{4}$ http://www.abc.net.au/news/2014-05-01/fact-file-what-tony-abbott-promised-on-tax/5420226
} 
level of discretion that the voters are willing to concede to the candidates is inversely related to their expected bias (where the bias is defined with respect to the median voter's preferences). In the main model the type structure is such that the variability and the expectation of the bias are interlinked. Therefore we introduce an example with a discrete type structure which allows for a comparative statics analysis on the variability of the bias for a given level of expected bias. The findings are ambiguous in determining the relation between the level of discretion and the uncertainty over the candidates' types; in some parametric regions an increase in the uncertainty over the candidate's types decreases the level of discretion that the voters' are willing to leave, as one would expect given that the voters are risk averse. In other parametric regions, however, the effect is the opposite and more uncertainty implies more discretion for the candidates; this is due to an insurance effect that the bounds of the set of promises exert: increasing the upper or lower bound (depending on whether the candidate is right-wing, or left-wing) has the effect of giving more discretion to good candidates' types, and not so much to bad candidates' types, who will hit the bound more often and will be constrained by it anyway.

Several papers analyse the causes of ambiguous statements in electoral campaigns. In his seminal work Shepsle (1972) analyses an electoral competition where a challenger can choose to be ambiguous in order to win against an incumbent who presents himself with a point policy. Shepsle shows that an ambiguous statement may be preferred to the median voter's preferred outcome, when many voters are risk loving. If the majority of voters are risk averse, Black's median voter theorem still applies, and ambiguity decreases the candidate's appeal at least as long as the majority of voters are risk averse. Page (1976) wrote a critique of Shepsle's model, stressing two problematic assumptions. In particular, he challenged the plausibility of individuals being risk loving, and candidates using lotteries. He viewed political statements not as distributions over the possible policies, but more as subsets of the possible outcomes, as appears from his writing: "This interpretation is supported by candidates' frequent proclamations of what they don't stand for [...] as if they were putting boundaries on their ambiguity, within a range of risk acceptance." ${ }^{5}$. Starting from this observation, he proposes a different explanation for the ambiguity of a politician's statement, based on the optimal allocation of emphasis and politicians' resources in the electoral campaign. Page considers multidimensional policies, and assumes that candidates can only partially control the emphasis that is put on their statements. In his setting, therefore, a candidate will find it optimal to

\footnotetext{
${ }^{5}$ Page (1976).
} 
be precise on non controversial issues, and to be vague on issues that are controversial, because any detailed statement on a controversial issue can be used against him by his opponent.

There are many recent works on vagueness in electoral campaigns. Most of the models present ambiguity as a candidate-driven phenomenon, which arises despite voters being ambiguity averse. These works are somehow tangential to the objective of this paper in which ambiguity arises rather directly from the joint uncertainty of the candidates and the voters. In particular, Glazer (1990) models ambiguity as generated by uncertainty on the bliss point of the median voter. Alesina and Cuckierman (1990) explain ambiguity as a device to conceal, at least partially, a politician's ideology; in their setting the incumbent can choose the level of ambiguity of his actions to avoid being locked in his ideological position, and losing votes in the subsequent election. Aragones and Neeman (2000) have similar results which exploit the candidate's tradeoff between committing to his most preferred strategy to increase his own utility ex post, and committing to the median voter's preferred point to maximize the probability of being elected; ambiguous promises arise as an optimal solution to this tradeoff. ${ }^{6}$ Meirowitz (2005) has a model of primaries in which candidates may choose strategically to remain ambiguous because they are aware of the fact that in the future they will become better informed on the preferences of the voters. Alesina and Holden (2008) model ambiguity in electoral campaigns as arising from a trade-off that the candidates face between moving towards the median voter's preferred outcome, to increase the chances of being elected, and taking a more extreme stand, to have more campaign contributions which help influencing the median voter's position. Laslier (2006) shows how candidates, when unable to target the political communication to a specific group, may benefit from the introduction of ambiguous platforms that may be interpreted as different policy positions by different recipients.

In this paper, the vagueness of the electoral statements arises in a natural way from a competitive delegation environment; the main objective of the paper is to see the consequences of this fairly standard way to generate ambiguity and to have a model which can be used to analyse the effect that several political variables have on the ambiguity levels of an electoral campaign. It is also worth noting that the form of ambiguity which features in the model is the one which Page found more appealing: ambiguous politicians simply put bounds on their own actions, and don't specify precisely which policy they will choose in the set of the possible ones.

\footnotetext{
${ }^{6}$ Other related works by Aragones includes Aragones et al. (2005) and Aragones and Postlewaite (2002).
} 
In the setting we analyse, both the candidates and the voters have a joint interest in discretion; this has similar effects to those described by Alonso and Matouschek (2008). They analyse the problem of an uninformed principal who wants to delegate an action to an informed agent; the authors characterise the optimal delegation as a function of the relationship between the agent's and the principal's preferences; the authors are interested in understanding the nature of the optimal delegation set, and derive, in a complete information setting, that interval delegation is optimal when agents' preferences are sufficiently aligned. Our model shows that this interval delegation result may hold also in an incomplete information setting, and it analyses in detail the effect of competition between candidates in the final choice of the set of promises.

As for many delegation models, voters can benefit from discretion. Indeed, there is now evidence that ambiguity might not be fully disliked by voters. Tomz and Van Houweling (2009) present an experimental setting in which they show that ambiguous candidates are not less likely to win the election even when they tie with the opponent in terms of the (expected) proposed policy; ambiguous candidates may even be more likely to win than unambigous ones when they belong to a specific party.

The paper is structured as follows. Section 2 introduces the model, the settings, the description of the state of the world, and of the agents' preferences. Section 3 analyses the equilibrium behavior. Section 4 shows an example of interesting comparative statics that may arise with different distribution of types and Section 5 concludes.

\section{The model}

We consider a political system characterized by two parties, $L$ and $R$, competing in one election. The electoral competition is based on one unidimensional policy decision $\widehat{p} \in \mathbb{R}$. The environment is characterised by a state of the world, $\omega$, which influences the optimal choice of the candidates and the preferences of the voters.

The electoral period is divided into three different stages:

Campaign stage: the candidates simultaneously announce their platforms. They can choose whether to announce a specific policy (a point) or a set of policies.

Voting stage: each voter casts her vote and a candidate is appointed.

Office stage: the state of the world $\omega$ is realized and the appointed candidate implements a policy.

The state of the world $\omega$ is unknown in the first two stages, and realised in the office stage. Both the voters and the candidates are assumed to learn the state of the world 
ex post, whereas ex ante they know that $\omega$ is a uniformly distributed random variable over $[-1,1]$.

During the campaign stage each candidate announces a closed set of policies $P$ to which his future implemented policy will belong. We assume that an appointed candidate cannot choose a policy outside the announced set $P \subseteq \mathbb{R}^{7}$

Voters are heterogeneous in terms of their preferred ideology and they care about the actual policy implemented. Each voter has a bliss point that depends on the realisation of the state of the world $(\omega)$ and on her ideology type $(t)$. So for a given state of the world voter $i$ 's preferred policy is given by $t_{i}+\omega$. The median voter's type $t_{m}$ is common knowledge and is normalised to zero. Calling $\widehat{p}$ the policy that is implemented, voter $i$ 's preferences are described by the following quadratic loss function:

$$
u_{i}(\widehat{p})=-\left(t_{i}+\omega-\widehat{p}\right)^{2}
$$

There are two types of candidates: candidate $L$, with ideological type $\tau_{L} \in[-z, 0]$, and candidate $R$, with ideological type $\tau_{R} \in[0, z]$. While the voters can distinguish whether a candidate is $L$ or $R$, they know only that for candidate $L$ ideological type $\tau_{L}$ is uniformly distributed on $[-z, 0]$, and for candidate $R$ on $[0, z]$ respectively.

Candidates get benefit $K$ from being in power and have zero utility otherwise; moreover, once they are in power, they suffer a disutility if they are not able to implement their preferred policy. Similarly to voters, for a given state of the world candidate $j \in\{L, R\}$ preferred policy is given by $\tau_{j}+\omega$. Therefore the utility function that represents the preferences of candidate $j \in\{L, R\}$ is:

$$
u_{j}(\widehat{p}, \omega)=\operatorname{Pr}\left(j \text { is elected } \mid P\left(\tau_{j}\right), \tau_{j}\right)\left[K-\left(\tau_{j}+\omega-\widehat{p}\right)^{2}\right],
$$

where $P\left(\tau_{j}\right)$ is candidate $j$ 's set of promises. A strategy for candidate $j$ is a function:

$$
s_{j}:\left(\tau_{j}, \omega\right) \mapsto\left(P\left(\tau_{j}\right), \widehat{p}\left(\tau_{j}, \omega\right)\right),
$$

with $j=L, R$. The function $s_{j}$ associates a set of policy promises and an implemented policy to every pair of ideology and realisation of the state of the world. Notice that the set of promises $P$ can depend only on the candidate's ideology because the state of the world is not known ex ante. Therefore, the choice of $P$ cannot reveal any information on the state of the world $\omega$.

\footnotetext{
${ }^{7}$ Evidence that the set of promises influences politicians' ex-post behavior is provided by Nicolò et al. (2014). Alternatively, the assumption of commitment over the set of promises can be interpreted as a reduced form of a multiperiod model in which the candidate is punished in future election periods for deviating from the promised set, as in Alesina (1988).
} 
We will focus only on pooling equilibria in pure strategies to understand the effects of uncertainty on candidates' discretion, disentangling them from the possible signalling effects. We assume that $K$ is large enough, so that the candidate behaves as if he had lexicographic preferences, caring first about being elected and then about which policy he is able to implement. Consider a candidate who changes his strategy from $\left(P_{1}, \hat{p}_{1}(\omega)\right)$ to $\left(P_{2}, \hat{p}_{2}(\omega)\right)$. This change can either affect the probability of being elected or not. If it does not affect it, the candidate will choose the new strategy if it allows him to implement his preferred policy more often ex post: if it does change his probability of being elected, the candidate will choose the set of promises that increases this probability, regardless of the constraint that this set may impose ex post on his actions. As the probability of being elected is either 0 or $\frac{1}{2}$ or 1 , then the associated change in probability is $\frac{1}{2}$. If $K$ is large enough, $\frac{1}{2} K$ is greater than any possible gain that can be obtained from changing set of promises.

\section{Equilibrium analysis}

We now analyze the equilibrium behavior. We prove that all equilibria have the same structure, and that they may differ only for the inclusion/exclusion from the set of promises of irrelevant policies, i.e. policies that are never chosen in equilibrium, regardless of the state of the world. The optimal set of promises is an interval, which constrains the candidate's behavior in the direction of his bias. For example, the rightwing candidate will optimally promise to implement a policy smaller than a threshold $p_{R}^{*}$. Given the promised set, the elected politician implements his preferred policy whenever possible, and the threshold policy otherwise. Proposition 1 fully characterizes the equilibrium behavior.

Proposition 1. Every equilibrium of the game has the same structure. Equilibria differ only in the optimal sets of promises which may include or exclude policies that are never implemented by the candidate, regardless of the state of the world. The following is one equilibrium of the game:

- Candidate $L$ promises $P\left(\tau_{L}\right)=\left[p_{L}^{*} ; 1\right]$, where $p_{L}^{*}=-1-\frac{1}{\sqrt{3}} z$ if $z>\frac{-2 \sqrt{3}}{\sqrt{3}+1}$, and is implicitly defined by the following equation otherwise

$$
\frac{\left(p_{L}^{*}-1\right)^{3}}{6 p_{L}^{*}}=z
$$

- Candidate $R$ promises $P\left(\tau_{R}\right)=\left[-1 ; p_{R}^{*}\right]$, where $p_{R}^{*}=1-\frac{1}{\sqrt{3}} z$ if $z \leq \frac{2 \sqrt{3}}{\sqrt{3}+1}$, and is 
implicitly defined by the following equation otherwise

$$
\frac{\left(p_{R}^{*}+1\right)^{3}}{6 p_{R}^{*}}=z .
$$

- Voters vote for candidate $L$ if $t_{i}<0$, for candidate $R$ if $t_{i}>0$ and randomize with equal probability if $t_{i}=t_{m}=0$.

- the elected politician implements $\tau_{j}+\omega$ if $\tau_{j}+\omega \in P\left(\tau_{j}\right)$ and $p_{j}^{*}$ otherwise.

We now provide the intuition for the equilibrium behavior at different stages of the game. The formal proof is contained in the Appendix.

Office stage. Assume that candidate $R$ has been elected after he promised $P\left(\tau_{R}\right)$ in the campaign stage. His optimal behaviour once elected, given that he is constrained to choose a policy in the set $P\left(\tau_{R}\right)$, is to choose $\tau_{R}+\omega$ if $\tau_{R}+\omega \in P\left(\tau_{R}\right)$, and to choose the policy $p \in P\left(\tau_{R}\right)$ that minimises the distance between $p$ and $\tau_{R}+\omega$ otherwise. Whenever a candidate is indifferent between two policies, we assume that he chooses it in a way that makes his best response function upper hemicontinuous. For example, if $P\left(\tau_{R}\right)=(-\infty, a] \cup[b, \infty)$ the candidate's best response will be to choose $\tau_{R}+\omega$ if $\tau_{R}+\omega$ $\in P\left(\tau_{R}\right)$, to choose $a$ if $a<\tau_{R}+\omega<\frac{a+b}{2}$ and to choose $b$ otherwise.

Voting stage. Voters in this model observe the set of promises made by each candidate and choose the one that maximises their expected utility $\mathbb{E} U_{i}\left(\tau_{j}, P\left(\tau_{j}\right)\right)$. Given that the voters are risk averse, as shown in Shepsle (1972), Black's median voter theorem applies; therefore the winning candidate is the one which maximizes the expected utility of the median voter $\mathbb{E} U_{m}\left(\tau_{j}, P\left(\tau_{j}\right)\right)$. As a tie-breaking rule, given that candidates are ex-ante symmetric, we assume that randomize with equal probability between candidates when indifferent.

Campaign stage. Candidates choose the optimal promise set during the campaign stage. First, such a policy set is necessarily an interval in the politician's policy space, as proved in the following Lemma.

Lemma 1. Every candidate's minimal optimal set of promises is an interval.

Proof. Let's prove the result for candidate $R$. Consider first the fact that, being a closed set and a subset of $\mathbb{R}$, the set of promises $P\left(\tau_{R}\right)$ is the finite union of closed intervals and points. Assume now that optimal policy promise set contains a hole such that it is given by $\left(\underline{p}_{R}, a\right] \cup\left[b, \bar{p}_{R}\right)$. Then the candidate will ex post choose $a$ for $\tau+\omega<\frac{a+b}{2}$, 
and $b$ otherwise. His best response is therefore upper hemicontinuous. This is a mean preserving spread compared to the situation in which the interval $(a, b)$ belongs to $P$ and the candidate chooses $\tau+\omega$ whenever $\tau+\omega \in(a, b)$. Since the median voter is risk averse, his utility is lower with the mean preserving spread; the same reasoning can be extended to $P\left(\tau_{R}\right)$ being an interval and a point, and a finite collection of points. The optimal set of promises is therefore an interval.

Second, the promise set is independent of the candidate's type, given that candidates behave as if they had lexicographic preferences and first maximize probability of being elected. Finally, we characterize the optimal set of promises by obtaining the lower and upper bounds of the interval $\left(\underline{p}_{R}\right.$ and $\bar{p}_{R}$ for candidate $\left.R\right)$. Also note that the optimal behaviour of the median voter is to vote for the candidate with a promise and expected type such that $\mathbb{E} U_{m}\left(\tau_{j}, P\left(\tau_{j}\right)\right)$ is the highest between the available candidates. Given this, the candidate will choose $\underline{p}_{R}$ and $\bar{p}_{R}$ to minimize disutility of the median voter. Solving the optimization problem leads to the optimal bounds described above.

Discretion in equilibrium. The first notable point is that the candidates can always retain some discretion for themselves, as the set of promises is always an interval with $\left|p_{j}^{*}\right| \in(0,1)$. This is due firstly to the fact that some information (whether the candidate is right-wing or left-wing) is always common knowledge. As a consequence, a right-wing candidate will always be allowed to implement policies that are ex-ante perceived as leftist, and viceversa, i.e., $[-1,0] \subset P\left(\tau_{R}\right)$ and $[0,1] \subset P\left(\tau_{L}\right)$. Moreover, ideological types are distributed uniformly over $[0, z]$ so that there is always a positive probability of good types (i.e., with a low bias), no matter how large $z$ is. This implies that $\left|p_{j}^{*}\right|>0$.

A second relevant observation is that the level of discretion that candidates can retain is monotonically decreasing in $z$, which is both a measure of the expected bias and the level of uncertainty of the candidate's types. Hence, this may be driven by the fact that voters dislike candidates that are more biased, and want to leave them less discretion, or by the fact that voters dislike candidates' types being highly uncertain. The following section sheds more light on the effects of expected bias and uncertainty through an example where candidates' types are finite.

The model could also be extended to allow for asymmetric distributions of candidates' types (e.g, $z_{l} \neq-z_{R}$ ). In this case there would be an extra effect on candidates' discretion which would favor the candidate who is less biased ex-ante, as he could retain more discretion, leaving the median voter indifferent between his set of promises and the opponent's one. 


\section{An example with a discrete distribution of types}

The model analyzed above does not allow us to disentangle the effects that a change in the variability of the candidate's bias has on the voters' propension to leave discretion to candidates. This is caused by the assumption on the distribution of types which, for the sake of tractability, does not allow us to change the variability of types without affecting the expected bias. Therefore, we consider a specific example where the expected bias of the candidate is independent from variability, to disentangle the two effects. Specifically, we assume that candidate $R$ has type $\tau-x$ with probability $\frac{1}{2}$ and type $\tau+x$ with probability $\frac{1}{2}$. Notice that it must be that $\tau>x$, since we are keeping the assumption that the candidate is a right candidate. In this case $\tau=\mathbb{E}\left(\tau_{R}\right)$, and the parameter $x$ characterizes the variability of $R$ 's type around the mean. The symmetric assumption holds for $L$, so that $\mathbb{E}\left(\tau_{L}\right)=-\tau$.

Proposition 2. Every equilibrium of the game has the same structure. Equilibria differ only in the optimal sets of promises which may include or exclude policies that are never implemented by the candidate, regardless of the state of the world. The following is one equilibrium of the game:

- Candidate L promises $P\left(\tau_{L}\right)=\left[p_{L}^{x} ; 1\right]$, where

$$
p_{L}^{x}= \begin{cases}-1+\left(\tau^{2}+x^{2}\right)^{\frac{1}{2}} & \text { if }-\tau>\frac{2(x-1)}{2-x} \\ -3+\left(8+(\tau-x)^{2}\right)^{\frac{1}{2}} & \text { if }-\tau \in\left[-1-x, \frac{2(x-1)}{2-x}\right] \\ 0 & \text { otherwise }\end{cases}
$$

- Candidate $R$ promises $P\left(\tau_{R}\right)=\left[-1 ; p_{R}^{x}\right]$, where

$$
p_{R}^{x}= \begin{cases}1-\left(\tau^{2}+x^{2}\right)^{\frac{1}{2}} & \text { if } \tau<\frac{2(1-x)}{2-x} \\ 3-\left(8+(\tau-x)^{2}\right)^{\frac{1}{2}} & \text { if } \tau \in\left[\frac{2(1-x)}{2-x}, 1+x\right] \\ 0 & \text { otherwise }\end{cases}
$$

- Voters vote for candidate $L$ if $t_{i}<0$, for candidate $R$ if $t_{i}>0$ and randomize with equal probability if $t_{i}=t_{m}=0$.

- the elected politician implements $\tau_{j}+\omega$ if $\tau_{j}+\omega \in P\left(\tau_{j}\right)$ and $p_{j}^{*}$ otherwise.

As in the previous analysis, the median voter chooses the candidate who proposes the set of promises that gives him the lower expected disutility. The winning candidate can still retain some discretion, in light of the fact that the median voter recognizes whether the candidate is right-winged or left-winged, that is, $[-1,0] \subset P\left(\tau_{R}\right)$ and $[0,1] \subset P\left(\tau_{L}\right)$ 
as before. If the expected type of the canidate is too biased, however, the set of promises may not include any additional element, i.e., $p_{j}^{x}$ may be equal to zero. This difference with the previous version of the model is due to the fact that types with very small biases may have zero probability (whenever $\tau-x$ is large), so that the median voter finds optimal to restrain the candidate in the direction of his bias. We are interested in evaluating separately the effects of changes in candidates expected type $(\tau)$ and variability $(x)$.

Effects of changes in the expected type. $(\tau)$ The expected bias, as in the baseline model, decreases the extent to which voters are willing to concede discretion to candidates. An increase in the expected bias of the candidate unambiguously decreases level of discretion that voters would like to leave him.

Effects of the introduction of uncertainty. The limit case in which $x=0$ gives us the complete information benchmark. Under the assumption that $x=0$ voters know that candidate $R$ 's type is $\tau$ and $p_{R}^{0}=1-\tau$ if $\tau \leq 1$ and 0 otherwise. If we move from a case in which the candidate's type is known to be $\tau$, to one in which it is expected to be $\tau(x>0)$ the level of discretion can increase or decrease, depending on the parameters. For example if $1<\tau<1.1, p=0$ if $x=0$, while $p=3-\left(8+(\tau-0.1)^{2}\right)^{\frac{1}{2}}>0$ if $x=0.1$ : more discretion is left to the candidate in the uncertain case. On the contrary, if $\tau<1, p=1-\tau$ in the case where the type of the candidate is known, which is larger than any bound that the candidate can achieve in the uncertain situation.

Comparative statics on the variability of the type. $(x)$ Notice moreover that this is the same effect that can be observed if $x$ increases when all the other parameters, $\tau$ in particular, are kept fixed. In this case an increase in $x$ affects both the two different solutions and the parametric regions in which they apply. In particular, a greater $x$ reduces $1-\left(\tau^{2}+x^{2}\right)^{\frac{1}{2}}$ and increases $3-\left(8+(\tau-x)^{2}\right)^{\frac{1}{2}}$; it as well increases $1+x$ moving some types $\tau$ from having an optimal solution of 0 to an optimal solution of $3-\left(8+(\tau-x)^{2}\right)^{\frac{1}{2}}$. The overall effect on $3-\left(8+(\tau-x)^{2}\right)$ is interesting because it is related to the insurance effect that this part of the solution has: when this is the optimal level of discretion, the more extreme candidate will hit the bound significantly more often than the less extreme candidate; an increase in $x$, in this case, makes the insurance effect even stronger, because the moderate candidate moves closer to the median voter's preferred policy, and the extreme candidate hits the bound more often. 


\section{Conclusions}

This paper analyses an electoral contest as a competitive delegation problem. The aim of the paper is to investigate, with a simple model, what are the characteristics of an election that favor the politicians' vagueness, for example the structure of the electoral system, or the nature of the candidates.

We show that the level of ambiguity of the electoral campaign when candidates' types are symmetrically distributed is related to the the median voters willingness to delegate, at least partially, policy decisions to an informed candidate, despite his bias. The candidates' discretion, however, depends negatively on their own expected bias with respect to the median voters preferred policy.

In a version of the model with discrete types, we are able to disentangle the effects of the expected bias fromthe effects of the variability of candidates' types. In this setup we show that an increase in the uncertainty about the candidates can increase or decrease the level of ambiguity of the system. Ambiguous promises have in fact a different effect on moderate and extreme candidates; they bind extreme candidates more often, thus providing some insurance for the median voter. This effect is more relevant when the candidates expected type is extreme; if this is the case, an increase in the uncertainty about the candidates type increases the likelihood that he is a less biased type, and the willingness of the median voter to give him discretion about the future policies, without worries about the more biased type, given the insurance effect of the promises.

\section{References}

Alesina A. and A. Cuckierman (1990), The politics of ambiguity, Quarterly Journal of Economics, 105, 829-850.

Alesina A. and R. Holden (2008), Ambiguity and extremism in elections, Working paper series W14143, NBER.

Alonso R. and N. Matouschek (2008), Optimal delegation, The Review of Economic Studies, 75, 259-293.

Aragones E. and Z. Neeman (2000), Strategic ambiguity in electoral competition, Journal of Theoretical Politics, 12, 183-204.

Aragones E. and A. Postlewaite (2002), Ambiguity in election games, Review of Economic Design, 7, 233-255.

Aragones E. T.R. Palfrey and A. Postlewaite (2005), Reputation and rethorics in elections, Working paper 05-027, PIER. 
Glazer A. (1990), The strategy of candidate ambiguity, American Political Science Review, 84, 237-241.

Laslier J.F. (2006), Ambiguity in electoral competition, Economics of Governance, 7, 195-210.

Meirowitz A. (2005), Informational party primaries and strategic ambiguity, Journal of Theoretical Politics, 17, 107-136.

Nicolò A., Corazzini, L., Maréchal, A., and S. Kube (2014), Elections and Deceptions: An Experimental Study on the Behavioral Effects of Democracy, American Journal of Political Science, 58, 579-592.

Page B.I. (1976), The theory of political ambiguity, American Political Science Review, 70, 742-752.

Shepsle K.A. (1972), The strategy of ambiguity: uncertanity and electoral competition, American Political Science Review, 66, 555-568.

Tomz M. and R.P. Van Houweling (2009), The electoral implications of candidate ambiguity, American Political Science Review, 103, 83-98. 


\section{Appendix}

\section{Proof of Proposition 1.}

This proof presents the results for candidate $\mathrm{R}$ only. The derivation for candidate $\mathrm{L}$ is symmetric.

Office stage. Assume that candidate $R$ has been elected after he promised $P\left(\tau_{R}\right)$ in the campaign stage. His optimal behaviour once elected, given that he is constrained to choose a policy in the set $P\left(\tau_{R}\right)$, is to choose $\tau_{R}+\omega$ if $\tau_{R}+\omega \in P\left(\tau_{R}\right)$, and to choose the policy $p \in P\left(\tau_{R}\right)$ that minimises the distance between $p$ and $\tau_{R}+\omega$ otherwise. Whenever a candidate is indifferent between two policies, we assume that he chooses it in a way that makes his best response function upper hemicontinuous. For example, if $P\left(\tau_{R}\right)=(-\infty, a] \cup[b, \infty)$ the candidate's best response will be to choose $\tau_{R}+\omega$ if $\tau_{R}+\omega$ $\in P\left(\tau_{R}\right)$, to choose $a$ if $a<\tau_{R}+\omega<\frac{a+b}{2}$ and to choose $b$ otherwise.

Voting stage. Voters in this model observe the set of promises made by each candidate and choose the one that maximises their expected utility $\mathbb{E} U_{i}\left(\tau_{j}, P\left(\tau_{j}\right)\right)$. Given that the voters are risk averse, as shown in Shepsle (1972), Black's median voter theorem applies; therefore the winning candidate is the one which maximizes the expected utility of the median voter $\mathbb{E} U_{m}\left(\tau_{j}, P\left(\tau_{j}\right)\right)$. As a tie-breaking rule, given that candidates are ex-ante symmetric, we assume that randomize with equal probability between candidates when indifferent.

Campaign stage. Candidates choose the optimal promise set during the campaign stage. First, as proved in Lemma 1 in Section 3, such a policy set is necessarily an interval in the politician's policy space. Second, the promise set is independent of the candidate's type, given that candidates behave as if they had lexicographic preferences and first maximize probability of being elected. So the major part of this proof deals with derivation of lower $-\underline{p}_{R}$ and upper $-\bar{p}_{R}$ bounds of that optimal interval.

Voters in this model observe the set of promises made by each candidate and choose the one that maximises their expected utility $\mathbb{E} U_{i}\left(\tau_{j}, P\left(\tau_{j}\right)\right)$. Given that the voters are risk averse, as shown in Shepsle (1972), Black's median voter theorem applies; therefore the winning candidate is the one which maximizes the expected utility of the median voter $\mathbb{E} U_{m}\left(\tau_{j}, P\left(\tau_{j}\right)\right)$. The median voter's optimal behaviour is to vote for the candidate with a promise and expected type such that $\mathbb{E} U_{m}\left(\tau_{j}, P\left(\tau_{j}\right)\right)$ is the highest between the available candidates. Given this, the candidate will choose $\underline{p}_{R}$ and $\bar{p}_{R}$ to minimize disutility of the median voter. The median voter's expected disutility when the candidate type is $\tau_{R} \sim \mathrm{U}[0 ; \mathrm{z}]$, state of the world is $\omega \sim \mathrm{U}[-1 ; 1]$, and the set of promises is $\mathrm{P}\left(\tau_{R}\right)$ $=\left[\underline{p}_{R} ; \bar{p}_{R}\right]$ is: 


$$
\begin{array}{r}
\mathbb{E} \mathrm{U}_{m}\left(\tau_{R}, P\left(\tau_{R}\right)\right)=\operatorname{Pr}\left[\omega+\tau \leq \underline{p}_{R}\right] \times \mathbb{E}\left[\left(\omega-\underline{p}_{R}\right)^{2} \mid \omega+\tau \leq \underline{p}_{R}\right]+ \\
\operatorname{Pr}\left[\omega+\tau>\underline{p}_{R}\right] \times \operatorname{Pr}\left[\omega+\tau \leq \bar{p}_{R}\right] \times \mathbb{E}\left[\tau^{2} \mid \omega+\tau>\underline{p}_{R}, \omega+\tau \leq \bar{p}_{R}\right]+ \\
\operatorname{Pr}\left[\omega+\tau>\bar{p}_{R}\right] \times \mathbb{E}\left[\left(\omega-\bar{p}_{R}\right)^{2} \mid \omega+\tau>\bar{p}_{R}\right]
\end{array}
$$

Without loss of generality we can derive the lower bound under the assumption that the upper bound is not binding. So we rewrite the expected disutility of the median voter when the upper bound of the promise set is not binding:

$$
\begin{array}{r}
\mathbb{E} \mathrm{U}_{m}\left(\tau_{R}, P\left(\tau_{R}\right)\right)=\operatorname{Pr}\left[\omega+\tau \leq \underline{p}_{R}\right] \times \mathbb{E}\left[\left(\omega-\underline{p}_{R}\right)^{2} \mid \omega+\tau \leq \underline{p}_{R}\right]+ \\
\operatorname{Pr}\left[\omega+\tau>\underline{p}_{R}\right] \times \mathbb{E}\left[\tau^{2} \mid \omega+\tau>\underline{p}_{R},\right]
\end{array}
$$

Now, to minimize the disutility in (4) one must take into account that conditional expectations and probabilities are based on the sum of two independent uniformly distributed random variables. The function is piece-wise differentiable on 6 intervals. But it is easy to note that the upper bound of the optimal promise set that we are looking for cannot exceed the possible state of world $\omega$, as the median voter wants the implemented policy to match $\omega$, so including policies outside $[-1,1]$ is never optimal. For example, if $\bar{p}_{R}>1$, candidate $\mathrm{R}$ will never implemented $p=\omega$ (not even when $\omega=1$ ). Noticing $\bar{p}_{R} \leq 1$ implies $\underline{p}_{R} \leq 1$.

This reduces the analysis to a function piece-wise differentiable on 3 relevant intervals. We tackle these intervals one by one below.

Region 1. $\underline{p}_{R} \leq-1$

This is the most straightforward case which transfers (2) into unconditional expectation.

$$
\mathbb{E}\left[\tau^{2}\right]=\int_{0}^{z} \int_{-1}^{1} \frac{\tau^{2}}{2 z} d w d t=\frac{z^{2}}{3}
$$

For any value of $\underline{p}_{R} \leq-1$ the expected utility of median voter takes on value of $\frac{z^{2}}{3}$, which is independent of $\underline{p}_{R}$.

The other two cases are more involved as both probabilities in (4) are not zero any more. Now the distinction between cases arises due to $\underline{p}_{R}+1$ being greater or lower than $z$. In particular note that $\operatorname{Pr}[\tau+\omega \leq p]$ corresponds to the area below the $-45^{\circ}$ line (see Figure below). It is easy to note that for $\omega=-1$ maximum possible value for $\tau$ is $\underline{p}_{R}+1$. Depending on whether it is greater or smaller than $z$, the expressions for calculating the area below the line will change. 


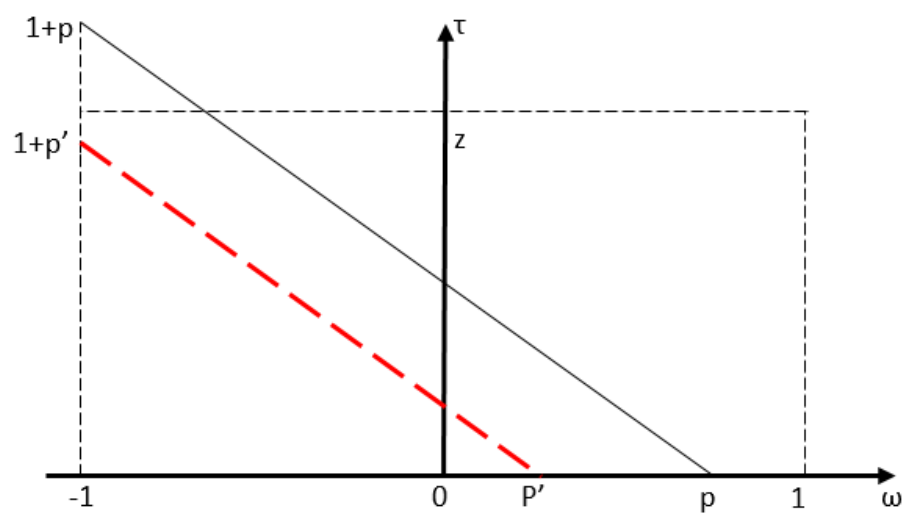

Region 2. $-1<\underline{p}_{R} \leq 1$ and $\underline{p}_{R}+1>z$

We calculate conditional probabilities and conditional expectations according to (4) for this particular case. To decrease the burden of notations in the expressions below we denote $\underline{p}_{R}=p$ and restore it at the end.

$$
\operatorname{Pr}[\tau+\omega \leq p]=\int_{0}^{z} \int_{-1}^{p-\tau} \frac{1}{2 z} d w d \tau=\frac{2 p+2-z}{4}
$$

We use properties of linear expectation to facilitate the derivation:

$$
\begin{array}{r}
\mathbb{E}\left[(\omega-p)^{2} \mid \tau+\omega \leq p\right]=\mathbb{E}\left[\omega^{2} \mid \tau+\omega \leq p\right]-2 p \mathbb{E}[\omega \mid \tau+\omega \leq p]+p^{2} \\
\mathbb{E}\left[\omega^{2} \mid \tau+\omega \leq p\right]=\frac{\int_{0}^{z} \int_{-1}^{p-\tau} \frac{\omega^{2}}{2 z} d w d \tau}{\int_{0}^{z} \int_{-1}^{p-\tau} \frac{1}{2 z} d w d \tau}=\frac{4 p^{3}-6 p^{2} z+4 p z^{2}-z^{3}+4}{12 p+12-6 z} \\
\mathbb{E}[\omega \mid \tau+\omega \leq p]=\frac{\int_{0}^{z} \int_{-1}^{p-\tau} \frac{\omega}{2 z} d w d \tau}{\int_{0}^{z} \int_{-1}^{p-\tau} \frac{1}{2 z} d w d \tau}=\frac{3 p^{2}-3 p z+z^{2}-3}{6 p+6-3 z} \\
\mathbb{E}\left[\tau^{2} \mid \tau+\omega>p\right]=\frac{\int_{0}^{z} \int_{p}^{1} \frac{\tau^{2}}{2 z} d w d \tau+\int_{p-z}^{p} \int_{p-w}^{z} \frac{\tau^{2}}{2 z} d \tau d w}{(2-2 p+z) / 4}=\frac{2\left(\frac{z^{3}}{4}-\frac{p z^{2}}{3}+\frac{z^{2}}{3}\right)}{2-2 p+z}
\end{array}
$$

Combining all these intermediate results we can rewrite the expected utility of the median voter as follows

$$
\mathbb{E} \mathrm{U}_{m}\left(\tau_{R}, P\left(\tau_{R}\right)\right)=\frac{2 p^{3}+6 p^{2}-2 p z^{2}+6 p+z^{3}+2 z^{2}+2}{12}
$$

The first order condition for the minimization problem yields $3 p^{2}+6 p-\left(z^{2}-3\right)=0$; the second order condition is satisfied when $p>-1$. The quadratic equation provides two candidates for optimal solution: $\frac{1}{3}(-\sqrt{3} z-3)$ and $\frac{1}{3}(-\sqrt{3} z+3)$. The first root 
doesn't belong to the eligible interval for $p$, whereas the second solution doesn't satisfy additional constraint of $\underline{p}_{R}+1>z$.

Region 3. $-1<\underline{p}_{R} \leq 1$ and $\underline{p}_{R}+1 \leq z$

Analogously to Region 2, we calculate conditional probabilities and conditional expectations.

$$
\begin{gathered}
\operatorname{Pr}[\tau+\omega \leq p]=\int_{0}^{p+1} \int_{-1}^{p-\tau} \frac{1}{2 z} d w d \tau=\frac{(p+1)^{2}}{4 z} \\
\mathbb{E}\left[\omega^{2} \mid \tau+\omega \leq p\right]=\frac{\int_{0}^{p+1} \int_{-1}^{p-\tau} \frac{w^{2}}{2 z} d w d \tau}{\int_{0}^{p+1} \int_{-1}^{p-\tau} \frac{1}{2 z} d w d \tau}=\frac{p^{4}+4 p+3}{6(p+1)^{2}} \\
\mathbb{E}[\omega \mid \tau+\omega \leq p]=\frac{\int_{0}^{p+1} \int_{-1}^{p-t} \frac{w}{2 z} d w d \tau}{\int_{0}^{p+1} \int_{-1}^{p-\tau} \frac{1}{2 z} d w d \tau}=\frac{p-2}{3} \\
\mathbb{E}\left[\tau^{2} \mid \tau+\omega>p\right]=\frac{\int_{-1}^{1} \int_{0}^{z} \frac{\tau^{2}}{2 z} d \tau d w-\int_{0}^{p+1} \int_{-1}^{p-\tau} \frac{t^{2}}{2 z} d w d \tau}{1-\frac{(p+1)^{2}}{4 z}}=\frac{\frac{z^{2}}{3}-\frac{(p+1)^{4}}{24 z}}{-\frac{p^{2}}{4 z}-\frac{p}{2 z}-\frac{1}{4 z}+1}
\end{gathered}
$$

Combining the intermediate results for this case we obtain:

$$
\mathbb{E} \mathrm{U}_{m}\left(\tau_{R}, P\left(\tau_{R}\right)\right)=\frac{p^{4}+4 p^{3}+6 p^{2}+4 p+4 z^{3}+1}{12 z}
$$

and F.O.C.s of the objective function in this interval yields optimal solution of $\underline{p}_{R}=$ -1 , which is again not in the admissible interval.

Combining all these observations we can conclude that there is a continuum of sets that are optimal for the candidate R: $P\left(\tau_{R}\right)=\left(\underline{p}_{R}, \bar{p}_{R}\right]$, with $\underline{p}_{R} \in(-\infty,-1]$. We choose $\underline{p_{R}}=-1$, linking the promised set to the true possible realizations of the state of the world. In this way we obtain a set of promises which is closed and bounded. Intuitively, the median voter has no incentive to impose a lower limit for the promises of right-winged candidate $R$. Even with the smallest bias, candidate $R$ will be never implementing policies to the left from the lowest possible state of the world: $\omega=-1$. One can obtain similar result for candidate $\mathrm{L}$ - a continuum of sets that are optimal: $P\left(\tau_{L}\right)=\left(\underline{p}_{L}, \bar{p}_{L}\right]$, with $\underline{p}_{L} \in[1, \infty)$. And again by matching the promised set to realizations of the state of the world $\underline{p}_{L}=1$.

At this point we know that candidate $\mathrm{R}$ will make a promise $\mathrm{P}\left(\tau_{R}\right)=\left[-1 ; p_{R}\right]$. Now the candidate has to choose the upper bound $\bar{p}_{R}$. To do this disutility in (3) is now rewritten knowing that the lower bound of the promise set is never binding:

$$
\begin{array}{r}
\mathbb{E} \mathrm{U}_{m}\left(\tau_{R}, P\left(\tau_{R}\right)\right)=\operatorname{Pr}\left[\omega+\tau>\bar{p}_{R}\right] \times \mathbb{E}\left[\left(\omega-\bar{p}_{R}\right)^{2} \mid \omega+\tau>\bar{p}_{R}\right]+ \\
\operatorname{Pr}\left[\omega+\tau \leq \bar{p}_{R}\right] \times \mathbb{E}\left[(\tau)^{2} \mid \omega+\tau \leq \bar{p}_{R}\right]
\end{array}
$$


Again considering all cases of the piecewise differentiable segments arising from the interactions of the model's parameters, there are two cases which are complying with our requirements. Note that from 6 possible intervals we rule out 3 cases when $\bar{p}_{R}>1$, as well as unconditional probability of $\bar{p}_{R}<-1$ is not relevant for upper bound.

Region 1. $-1<\bar{p}_{R} \leq 1$ and $\bar{p}_{R}+1 \leq z$

$$
\mathbb{E} \mathrm{U}_{m}\left(\tau_{j}, P\left(\tau_{j}\right)\right)=\frac{1}{12}\left(-2 p^{3}+6 p^{2}+2 p\left(z^{2}-3\right)-z^{3}+2 z^{2}+2\right)
$$

The F.O.C. in this case is given by $\frac{1}{6}\left(-3 p^{2}+6 p+z^{2}-3\right)=0$. Solving the F.O.C for $p$ we obtain candidates for optimal solution: $p_{R}^{*}=1 \pm \frac{1}{\sqrt{3}} z$. The second order condition for a minimum is given by $\frac{1}{6}(6-6 p)>0$ and satisfied whenever $p<1$. This implies $p_{R}^{*}=1-\frac{1}{\sqrt{3}} z$ with the constraint that $z \leq \frac{2 \sqrt{3}}{\sqrt{3}+1}$. The later stems from the second condition put on Region 1: $\bar{p}_{R}+1 \leq z$.

\section{Region 2. $-1<\bar{p}_{R} \leq 1$ and $\bar{p}_{R}+1>z$}

$$
\mathbb{E} \mathrm{U}_{m}\left(\tau_{j}, P\left(\tau_{j}\right)\right)=\bar{p}_{R}^{2}-\frac{\left(\bar{p}_{R}+1\right)^{4}}{12 z}+\frac{1}{3}
$$

For this case F.O.C. implicitly defines optimal $\bar{p}_{R}: \frac{(p+1)^{3}}{6 p}=z$. In order condition of $\bar{p}_{R}+1>z$ to be satisfied following must hold: $z>\frac{2 \sqrt{3}}{\sqrt{3}+1}$. Second order condition for a minimum is satisfied when $2-\frac{(p+1)^{2}}{z}>0$. Finally note that $p_{R}$ cannot be negative as this would imply negative $z$. So optimal upper bound is always greater than zero.

So the overall solution provides the optimal promise set for Candidate $R-P\left(\tau_{R}\right)=$ $\left[-1 ; p_{R}^{*}\right]$, where $p_{R}^{*}=1-\frac{1}{\sqrt{3}} z$ if $z \leq \frac{2 \sqrt{3}}{\sqrt{3}+1}$, and is implicitly defined by $\frac{\left(p_{R}^{*}+1\right)^{3}}{6 p_{R}^{*}}=z$ if $z>\frac{2 \sqrt{3}}{\sqrt{3}+1}$. 


\section{Proof of Proposition 2.}

The median voter's utility function is as follows:

$$
\begin{array}{r}
\mathbb{E} \mathrm{U}_{m}(\tau, x, P(\tau, x))=\frac{1}{2}\left\{\operatorname{Pr}[\omega+\tau-x>p] \mathbb{E}\left[(\omega-p)^{2} \mid \omega+\tau-x>p\right]+\right. \\
\left.\operatorname{Pr}[\omega+\tau-x \leq p] \mathbb{E}\left[(\tau-x)^{2} \mid \omega+\tau-x \leq p\right]\right\}+ \\
\frac{1}{2}\left\{\operatorname{Pr}[\omega+\tau+x>p] \mathbb{E}\left[(\omega-p)^{2} \mid \omega+\tau+x>p\right]+\right. \\
\left.\operatorname{Pr}[\omega+\tau+x \leq p] \mathbb{E}\left[(\tau+x)^{2} \mid \omega+\tau+x \leq p\right]\right\}
\end{array}
$$

Recall that $x<1$, is subject to $p_{R} \in[0,1]$ and $\tau>x$. The objective function is a piecewise function defined over the following intervals.

Region 1: $p<\tau-x-1$. Under this restriction second and fourth addendum in (20) above are equal to 0 , whereas first and third are equal to 1 . Hence we obtain the unconditional expectations: $\frac{1}{2} \mathbb{E}(\omega-p)^{2}+\frac{1}{2} \mathbb{E}(\omega-p)^{2}=\frac{1}{3}+p^{2}$. It is immediate to note that the optimal promise from the median voter's point of view is $p=0$.

Region 2: $p \in[\tau-x-1, \tau+x-1]$. Under this restriction third addendum in (20) is equal to 1 , whereas fourth is equal to 0 . So the expected utility can be represented as: $\frac{1}{2} \frac{\tau-x+1-p}{2} \mathbb{E}\left[(\omega-p)^{2} \mid \omega+\tau-x>p\right]+\frac{1}{2} \frac{p-\tau+x+1}{2} \mathbb{E}\left[(\tau-x)^{2} \mid \omega+\tau-x \leq\right.$ $p]+\frac{1}{2} \mathbb{E}(\omega-p)^{2}$. Expanding also the expressions for conditional expectations we obtain objective function that the median voter would like to see minimized:

$$
\frac{1}{4}\left[\frac{1}{3}-\frac{p^{3}}{3}-\frac{2}{3}(\tau-x)^{3}-p+p^{2}+1(\tau-x)^{2}+p(\tau-x)^{2}\right]+\frac{1}{2}\left[\frac{1}{3}+p^{2}\right],
$$

therefore the first order condition is

$$
-p^{2}-1+\tau^{2}+x^{2}-2 \tau x+6 p=0,
$$

which gives us the following candidates for the solution:

$$
p=3 \pm\left(8+(\tau-x)^{2}\right)^{\frac{1}{2}}
$$

The second order condition says that this is a minimum provided that $-2 p+6>$ 0 , that is provided that $p<3$. Therefore the only candidate to be a minimum in this region is $p=3-\left(8+(\tau-x)^{2}\right)^{\frac{1}{2}}$; we need to check if $3-\left(8+(\tau-x)^{2}\right)^{\frac{1}{2}} \in$ $[\tau-x-1, \tau+x-1]$. 
- $3-\left(8+(\tau-x)^{2}\right)^{\frac{1}{2}}>\tau-x-1$ whenever $4-(\tau-x)>\left(8+(\tau-x)^{2}\right)^{\frac{1}{2}}$. If $\tau-x>4$ the inequality is never satisfied. If $\tau-x<4$ both sides are positive, so the inequality holds if and only if $(4-(\tau-x))^{2}>8+(\tau-x)^{2}$, that is iff $16+(\tau-x)^{2}-8(\tau-x)>8+(\tau-x)^{2}$. The inequality reduces to $1-\tau+x>0$, which is satisfied when $\tau<1+x$.

- $3-\left(8+(\tau-x)^{2}\right)^{\frac{1}{2}}<\tau+x-1$ whenever $4-\tau-x<\left(8+(\tau-x)^{2}\right)^{\frac{1}{2}}$. If $\tau+x<4$ the inequality is always satisfied, because the left handside is negative and the right one is positive. If $\tau+x>4$ the inequality holds if and only if $16+\tau^{2}+x^{2}+2 \tau x-8 \tau-8 x<8+\tau^{2}+x^{2}+2 \tau x$, that is, iff $8 q^{2}+4 \tau x-8 q(\tau+x)<0$. The inequality is satisfied when $\tau>\frac{2(1-x)}{2-x}$. Notice that $\frac{2(1-x)}{2-x}<4-x$, which implies that the inequality is satisfied for every $\tau$.

This implies that whenever $\tau<1+x$, the minimum of the objective function in this region is attained at $p=3-\left(8+(\tau-x)^{2}\right)^{\frac{1}{2}}$, while when $\tau>1+x$, the minimum is attained at $p=\tau-x-1$.

The expected disutility at $3-\left(8+(\tau-x)^{2}\right)^{\frac{1}{2}}$ is:

$$
\frac{1}{4}\left[-50+18\left(8+(\tau-x)^{2}\right)^{\frac{1}{2}}-2(\tau-x)^{2}-\frac{2}{3}(\tau-x)^{3}\right] .
$$

Region 3: $p \in[\tau+x-1, \tau-x+1]$. In this region the objective is to minimise:

$$
\begin{aligned}
& \frac{1}{2}\left[\frac{1}{2}\left(\frac{1}{3}-\frac{p^{3}}{3}-\frac{2}{3}(\tau-x)^{3}-p+p^{2}+(\tau-x)^{2}+p(\tau-x)^{2}\right)\right] \\
& +\frac{1}{2}\left[\frac{1}{2}\left(\frac{1}{3}-\frac{p^{3}}{3}-\frac{2}{3}(\tau+x)^{3}-p+p^{2}+(\tau+x)^{2}+p(\tau+x)^{2}\right)\right] .
\end{aligned}
$$

The first order condition is $-p^{2}-1+2 p+\tau^{2}+x^{2}=0$, therefore the possible solutions are $p=1 \pm\left(\tau^{2}+x^{2}\right)^{\frac{1}{2}}$. The second order condition for a minimum requires $-2 p+2>0$, that is $p<1$, hence the solution in this region is $p=1-\left(\tau^{2}+x^{2}\right)^{\frac{1}{2}}$, which belongs to $[\tau+x-1, \tau-x+1]$ when $\tau \leq \frac{2(1-x)}{2-x}$ and $\tau<2-x$.

The expected disutility at $p=1-\left(\tau^{2}+x^{2}\right)^{\frac{1}{2}}$ is

$$
\left[-\frac{1}{3} \tau^{3}+x^{2}+\tau^{2}-\frac{1}{3}\left(\tau^{2}+x^{2}\right)^{\frac{3}{2}}-\tau x^{2}\right]
$$

Region 4: $p \in[\tau-x+1, \tau+x+1]$. In this region the objective is to minimise

$$
\frac{1}{2}\left[\frac{1}{2}\left(\frac{1}{3}-\frac{p^{3}}{3}-\frac{2}{3}(\tau+x)^{3}-p+p^{2}+(\tau+x)^{2}+p(\tau+x)^{2}\right)\right]+\frac{1}{2}\left[(\tau-x)^{2}\right] .
$$


The first order condition is: $-p^{2}-1+2 p+\tau^{2}+x^{2}+2 \tau x=0$, implying that the possible solutions are $p=1 \pm(\tau+x)$. The second order condition imposes that $p<1$, so the only candidate for a local minimum is $1-(\tau+x)$, while $1+\tau+x$ is a local maximum. However $1-(\tau+x)$ does not belong to this region. Therefore the minimum in this region is attained at $\tau-x+1^{8}$, where the expected disutility is:

$$
-\frac{1}{3} x^{3}+\tau^{2}+x^{2}
$$

Region 5: $p>\tau+x+1$. In this region the objective function is $\frac{1}{2}\left[(\tau-x)^{2}\right]+$ $\frac{1}{2}\left[(\tau+x)^{2}\right]$ which does not depend on $p$. Any $p$ in the region is equivalent to the median voter and gives expected disutility $\frac{1}{2}\left[(\tau-x)^{2}\right]+\frac{1}{2}\left[(\tau+x)^{2}\right]=\tau^{2}+x^{2}$.

\section{Overall solution.}

1. Solutions in region 4 and 5 are never global optima: a solution in region 5 is always dominated by a solution in region 4 ;

2. Whenever $\tau>1+x$ the solution is $p=0$, because all the other solutions collapse to $p=0$;

3. When $\frac{2(1-x)}{2-x}<\tau<1+x$ the minimum is at $3-\left(8+(\tau-x)^{2}\right)^{\frac{1}{2}}$, because region 1 is not relevant $(\tau-x-q<0)$ and the solution in region 3 belongs to region 2 as well (we know by the optimisation in region 2 that $3-\left(8+(\tau-x)^{2}\right)^{\frac{1}{2}}$ is better than $\tau+x-1$.

4. When $\tau<\frac{2(1-x)}{2-x}$ the minimum is at $1-\left(\tau^{2}+x^{2}\right)^{\frac{1}{2}}$ because the solution in region 2 collapses to $\tau+x-1$ which is also a solution in region 3 .

All this taken into account, the solution has the following shape:

$$
p_{R}^{x}= \begin{cases}1-\left(\tau^{2}+x^{2}\right)^{\frac{1}{2}} & \text { if } \tau \leq \frac{2(1-x)}{2-x} \\ 3-\left(8+(\tau-x)^{2}\right)^{\frac{1}{2}} & \text { if } \tau \in\left[\frac{2(1-x)}{2-x}, 1+x\right] \\ 0 & \text { otherwise. }\end{cases}
$$

\footnotetext{
${ }^{8}$ Over the interval $[\tau-x+1, \tau+x+1]$ the function in question is concave, with is local maximum at $\tau+x+1$. Therefore the local minimum is at the other extreme of the interval.
} 